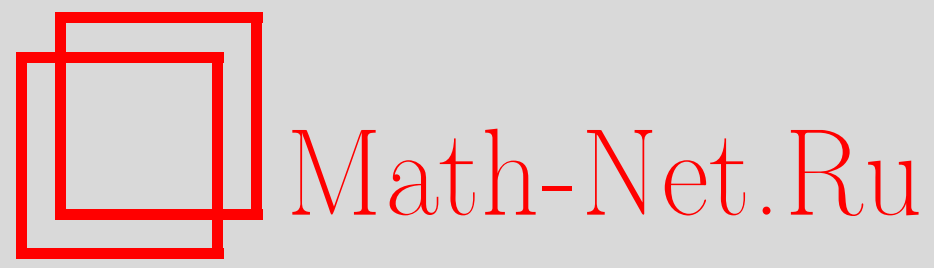

С. В. Архипов, О преобразовании Лапласа однородных функций в $\mathbb{R}^{n}$, Вестник ТвГУ. Серия: Прикладная математика, 2020, выпуск 1, 60-71

DOI: https://doi.org/10.26456/vtpmk555

Использование Общероссийского математического портала Math-Net.Ru подразумевает, что вы прочитали и согласны с пользовательским соглашением

http://www.mathnet.ru/rus/agreement

Параметры загрузки:

IP : 18.209 .158 .208

26 апреля 2023 г., 10:24:30 
УДК $517.521 .5,517.442$

\section{О ПРЕОБРАЗОВАНИИ ЛАПЛАСА ОДНОРОДНЫХ ФУНКЦИЙ $\mathbf{B} \mathbb{R}^{n}$}

Архипов С.В.

Тверской государственный университет, г. Тверь

Поступила в редакиию 20.01.2020, после переработки 01.03.2020.

В одномерном случае преобразование Лапласа степенных функций относится $\mathrm{K}$ табличным интегралам. Многомерным аналогом степенных функций являются однородные функции $\theta(\tau)|t|^{\alpha}$, $\tau \in S^{n-1}=\left\{t \in \mathbb{R}^{n}:|t|=1\right\}$, где $\alpha$ - степень однородности, а $\theta(\tau)$ - функция на единичной сфере. Для сходимости интеграла необходимо рассматривать область $\gamma$, лежащую внутри некоторой полусферы. При вычислении преобразования Лапласа однородных функций нужно вывести явное представление. Это достигается применением Фурье - анализа на сфере, а также суммированием интегралов с использованием ядра преобразования Фурье, позволяющего построить простое аналитическое продолжение гипергеометрических функций, появляющихся при вычислениях. В статье получены формулы для преобразования Лапласа однородных функций, у которых $\theta(\tau)$ принадлежит различным функциональным пространствам на единичной сфере с носителем $\gamma$.

Ключевые слова: многомерное преобразование Лапласа, однородные функции, сферические гармоники, ряд Фурье-Лапласа.

Вестник ТвГУ. Серия: Прикладная математика. 2020. № 1. С. 60-71. https://doi.org/10.26456/vtpmk555

\section{Введение}

Будем использовать следующие обозначения: $\Gamma$ - выпуклый острый телесный конус в $\mathbb{R}^{n}$, имеющий вершину в начале координат. Сопряженный к $Г$ конус, являющийся также острым, определим как $\Gamma^{*}=\{x:(x, t) \geqslant 0, t \in \Gamma\}$. Кроме того $C=\operatorname{int} \Gamma^{*}, \gamma=p r \Gamma-$ проекция конуса $\Gamma$ на единичную сферу $S^{n-1}$.

Преобразование Лапласа тесно связано с тауберовыми теоремами. В книге [2] они доказываются в достаточно общей ситуации, связанной с обобщенными функциями. В статье рассматриваются однородные функции, определенные в конусе $\Gamma$ и вычисляется их преобразование Лапласа

$$
L \theta(\tau)|t|^{\alpha}=\int_{\Gamma} \exp (-(x, t)) \theta(\tau)|t|^{\alpha} d t, \quad x \in C .
$$

Для преобразований применяется Фурье-анализ на единичной сфере (см. [1,6, $9,10])$, в частности для функции $\theta(\tau)$, заданной на $\gamma$, используется продолжение 
четным образом на $-\gamma$. Тогда разложение в ряд Фурье-Лапласа будет иметь вид:

$$
\theta(\tau)=\sum_{l=0}^{\infty} \sum_{j=1}^{d(l)} \theta_{2 l, j} Y_{2 l, j}(\tau),
$$

где $Y_{l j}(\tau)$ - сферические гармоники, образующие ортонормированный базис на $S^{n-1}, l=0,1,2, \ldots, j=1,2, \ldots, d(l)$, a $d(l)$ - размерность пространства сферических гармоник порядка $l$.

Коэффициенты разложения определяются по формуле

где

$$
\theta_{2 l, j}=\int_{S_{+}^{n-1}(\xi)} \theta(\tau) I(\gamma) Y_{2 l, j}(\tau) d \tau,
$$

$$
S_{+}^{n-1}(\xi)=\left\{\tau \in S^{n-1}:(\xi, \tau)>0\right\} .
$$

Сформулируем основные результаты.

Теорема 1. Пусть $\theta(\tau) \in C^{\infty}\left(S^{n-1}\right)$ - пространству бесконечно дифференцируемых функиий с носителем $\gamma$. Тогда при $\alpha>-n$ u $\alpha+n \neq 1,3,5, \ldots$

$$
\begin{aligned}
& \int_{\Gamma} \exp (-(x, t)) \theta(\tau)|t|^{\alpha} d t= \\
& \quad=\frac{2^{\alpha+n-1} \pi^{(n-1) / 2}}{|x|^{\alpha+n} \cos \pi\left(\frac{\alpha+n}{2}\right)} \sum_{l, j}(-1)^{l} \frac{\Gamma\left(l+\frac{\alpha+n}{2}\right)}{\Gamma\left(l-\frac{\alpha}{2}\right)} \theta_{2 l, j} Y_{2 l, j}(\xi) .
\end{aligned}
$$

Замечание 1 . Отметим, что при четных $\alpha$ суммирование ряда по $l$ начинается с $\frac{\alpha}{2}+1$.

Поскольку преобразование Лапласа представляется с помощью рядов ФурьеЛапласа с мультипликатором, то порядок однородной функции связан с гладкостью функции $\theta(\tau)$ на сфере.

Теорема 2. Пусть $\theta(\tau) \in L_{2}^{r}\left(S^{n-1}\right)$ - пространству дифференцируемых функиий на сфере $S^{n-1}$ с носителем $\gamma$, чви производные до порлдка г существуют и принадлежат пространству $L_{2}\left(S^{n-1}\right)$ суммируемых с квадратом функиий. Тогда (2) справедливо при $r \geqslant \max (0, \alpha+n / 2) u \alpha>-n$, а также при $\alpha+n \neq 1,3,5, \ldots$

При вычислении интегралов возникает необходимость устранения особенностей в нуле и единице. Для этого используют интегрирование по двойной петле, позволяющее построить аналитическое продолжение по параметрам (см. [4], п. 1.2). Нам понадобятся две формулы с интегралами по двойной петле, устраняющие ограничения на параметры. Первая из них имеет вид

$$
\int_{(0)}^{(1)} x^{\alpha-1}(1-x)^{\beta-1} d x=\frac{\Gamma(\alpha) \Gamma(\beta)}{\Gamma(\alpha+\beta)}
$$

и выполняется для всех $\alpha$ и $\beta$, за исключением случаев, когда $\alpha$ или $\beta$ равны $0,-1,-2, \ldots$ (см. [4], п. 1.3). 
Кроме того необходимо расширить действие формулы 5.2.3(2) из [5]. Применяя также интегрирование по двойной петле, охватывающей точки $z_{1}=0$ и $z_{2}=1$ в комплексной плоскости, придем к соотношению

$$
\begin{aligned}
{ }_{3} F_{2}\left(\beta, \alpha_{1}, \alpha_{2} ; \beta+\sigma, \gamma_{1} ; z\right)= & \frac{\Gamma(\beta+\sigma)}{\Gamma(\beta) \Gamma(\sigma)} \cdot \\
& \cdot \int_{(0)}^{(1)} t^{\beta-1}(1-t)^{\sigma-1}{ }_{2} F_{1}\left(\alpha_{1}, \alpha_{2} ; \gamma_{1} ; z t\right) d t
\end{aligned}
$$

справедливому для всех значений $\beta$ и $\sigma$ для которых функции от них имеют смысл.

\section{1. Доказательство теорем}

Доказательство теоремы 1. Запишем формулу для преобразования Лапласа однородной функции порядка $\alpha$ и сделаем замену переменной $r=|x||t|$ :

$$
L \theta(\tau)|t|^{\alpha}=\int_{\Gamma} e^{-(x, t)} \theta(\tau)|t|^{\alpha} d t=\frac{1}{|x|^{\alpha+n}} \int_{\gamma} \int_{0}^{\infty} e^{-(\xi, \tau) r} \theta(\tau) r^{\alpha+n-1} d r d \tau .
$$

В дальнейшем для получения конечного результата применим суммирование интегралов с использованием ядра преобразования Фурье, которое приводит к простому аналитическому продолжению. Условие $(\xi, \tau)>0, \xi \in p r C, \tau \in \gamma$ обеспечивает равномерную сходимость интеграла по $r$. Поэтому взятие предела по $\varepsilon$ не меняет значения преобразования Лапласа:

$$
L \theta(\tau)|t|^{\alpha}=\lim _{\varepsilon \rightarrow 0} \frac{1}{|x|^{\alpha+n}} \int_{\gamma} \int_{0}^{\infty} e^{-(\xi, \tau) r} e^{-i \varepsilon r} \theta(\tau) r^{\alpha+n-1} d r d \tau .
$$

Применим во внутреннем интеграле формулу (3.381.4) [3]. Тогда

$$
\begin{aligned}
L \theta(\tau)|t|^{\alpha}=\frac{\Gamma(\alpha+n)}{|x|^{\alpha+n}} \lim _{\varepsilon \rightarrow 0} & \int_{\gamma} \frac{\theta(\tau)}{((\xi, \tau)+i \varepsilon)^{\alpha+n}} d \tau= \\
& =\frac{1}{|x|^{\alpha+n}} \lim _{\varepsilon \rightarrow 0} \frac{\Gamma(\alpha+n)}{(i \varepsilon)^{\alpha+n}} \int_{\gamma} \frac{\theta(\tau)}{((\xi, \tau) /(i \varepsilon)+1)^{\alpha+n}} d \tau .
\end{aligned}
$$

Вычислим отдельно выражение под знаком предела в (5), обозначив его через $I$. Применим в подынтегральной функции биномиальное разложение в ряд, справедливое при $|x|<1$ :

$$
(1+x)^{-\nu}=\sum_{k=0}^{\infty}(-1)^{k} \frac{\Gamma(\nu+k)}{\Gamma(\nu) \Gamma(k+1)} x^{k} .
$$

Предполагая, что $\varepsilon>1$, будем иметь

$$
\begin{gathered}
I=\frac{\Gamma(\alpha+n)}{(i \varepsilon)^{\alpha+n}} \int_{\gamma} \frac{\theta(\tau)}{((\xi, \tau) /(i \varepsilon)+1)^{\alpha+n}} d \tau= \\
\quad=\frac{1}{(i \varepsilon)^{\alpha+n}} \int_{\gamma} \sum_{k=0}^{\infty}(-1)^{k} \frac{\Gamma(\alpha+n+k)}{\Gamma(k+1)}((\xi, \tau) /(i \varepsilon))^{k} \theta(\tau) d \tau .
\end{gathered}
$$


Поменяем местами знаки суммирования и интегрирования. Обоснование законности этого проведем позже.

$$
I=\frac{1}{(i \varepsilon)^{\alpha+n}} \sum_{k=0}^{\infty}(-1)^{k} \frac{\Gamma(\alpha+n+k)}{(i \varepsilon)^{k} \Gamma(k+1)} \int_{\gamma}(\xi, \tau)^{k} \theta(\tau) d \tau .
$$

Подставим разложение $(1)$ для $\theta(\tau)$ в ряд Фурье-Лапласа по сферическим гармоникам под знак интеграла (вынесение знака суммы по $l$ обеспечивается равномерной сходимостью ряда):

$$
I=\frac{1}{(i \varepsilon)^{\alpha+n}} \sum_{k=0}^{\infty}(-1)^{k} \frac{\Gamma(\alpha+n+k)}{(i \varepsilon)^{k} \Gamma(k+1)} \cdot \sum_{l, j} \theta_{2 l, j} \int_{S_{+}^{n-1}(\xi)}(\xi, \tau)^{k} Y_{2 l, j}(\tau) d \tau
$$

Для вычисления интеграла воспользуемся формулой (1.19) из [7] :

$$
\begin{gathered}
\int_{S^{n-1}}|(\xi, \tau)|^{k} Y_{2 l, j}(\tau) d \tau=2 \int_{S_{+}^{n-1}(\xi)}(\xi, \tau)^{k} Y_{2 l, j}(\tau) d \tau= \\
=\frac{2^{1-k} \pi^{n / 2} \Gamma(k+1)}{\Gamma\left(\frac{2 l+n+k}{2}\right) \Gamma\left(1+\frac{k-2 l}{2}\right)} Y_{2 l, j}(\xi),
\end{gathered}
$$

причем при $k-2 l=-2,-4,-6, \ldots$ интеграл равен 0 . Применяя последнюю формулу в (6), запишем

$$
I=\frac{1}{(i \varepsilon)^{\alpha+n}} \sum_{k=0}^{\infty}(-1)^{k} \sum_{l, j} \theta_{2 l, j} Y_{2 l, j}(\xi) \frac{2^{-k} \pi^{n / 2} \Gamma(\alpha+n+k)}{(i \varepsilon)^{k} \Gamma\left(\frac{2 l+n+k}{2}\right) \Gamma\left(1+\frac{k-2 l}{2}\right)} .
$$

Вычислим отдельно ряды по четным и нечетным $k$, обозначив их соответственно $I_{\text {чет }}$ и $I_{\text {нечет }}$. Тогда

$$
I_{\text {чет }}=\frac{\pi^{n / 2}}{(i \varepsilon)^{\alpha+n}} \sum_{k=0}^{\infty} \sum_{l, j} \theta_{2 l, j} Y_{2 l, j}(\xi) \frac{2^{-2 k} \Gamma(\alpha+n+2 k)}{(i \varepsilon)^{2 k} \Gamma\left(\frac{2 l+n+2 k}{2}\right) \Gamma(1+k-l)} .
$$

Отметим, что если $k-l=-1,-2,-3, \ldots$, то $1 / \Gamma(1+k-l)=0$. Введем новый индекс суммирования: $K=k-l$. Перепишем сумму ряда по $k$ и поменяем местами знаки суммирования:

$$
I_{\text {чет }}=\frac{\pi^{n / 2}}{(i \varepsilon)^{\alpha+n}} \sum_{l, j} \theta_{2 l, j} Y_{2 l, j}(\xi) \sum_{K=0}^{\infty} \frac{2^{-(2 K+2 l)} \Gamma(2(K+l+(\alpha+n) / 2))}{(i \varepsilon)^{2 K+2 l} \Gamma\left(\frac{4 l+2 K+n}{2}\right) \Gamma(K+1)} .
$$

Преобразуем гамма-функцию в числителе по формуле двойного аргумента:

$$
I_{\text {чет }}=\frac{\pi^{(n-1) / 2} 2^{\alpha+n-1}}{(i \varepsilon)^{\alpha+n}} \sum_{l, j}(-i / \varepsilon)^{2 l} \theta_{2 l, j} Y_{2 l, j}(\xi) .
$$




$$
\cdot \sum_{K=0}^{\infty}(-i)^{2 K} \frac{\Gamma((K+l+(\alpha+n) / 2)) \Gamma((K+l+(\alpha+n+1) / 2))}{\varepsilon^{2 K} \Gamma\left(K+2 l+\frac{n}{2}\right) \Gamma(K+1)} .
$$

При $\varepsilon>1$ второй ряд является гипергеометрическим. Он равномерно и абсолютно сходится, когда модуль аргумента меньше единицы. Используя обозначение гипергеометрического ряда, получаем

$$
\begin{aligned}
I_{\text {чет }}=\frac{\pi^{(n-1) / 2} 2^{\alpha+n-1}}{(i \varepsilon)^{\alpha+n}} . & \\
\cdot \sum_{l, j} \frac{(-1)^{l}}{\varepsilon^{2 l}} \theta_{2 l, j} Y_{2 l, j}(\xi) & \frac{\Gamma(l+(\alpha+n) / 2) \Gamma(l+(\alpha+n+1) / 2)}{\Gamma\left(2 l+\frac{n}{2}\right)} . \\
& \quad \cdot{ }_{2} F_{1}\left(l+\frac{\alpha+n}{2}, l+\frac{\alpha+n+1}{2} ; 2 l+\frac{n}{2} ;-\frac{1}{\varepsilon^{2}}\right) .
\end{aligned}
$$

Очевидно, что при $\varepsilon>1-1 / \varepsilon^{2} \in(-1,0)$. Осуществим аналитическое продолжение гипергеометрического ряда в комплексную плоскость с разрезом по лучу $(1, \infty)$ с помощью преобразования $w=z /(z-1)$. (см. [3], (9.131.1)):

$$
{ }_{2} F_{1}(a, b ; c ; z)=(1-w)^{a} \cdot{ }_{2} F_{1}(a, c-b ; c ; w) .
$$

Тогда имеем

$$
\begin{aligned}
& I_{\text {чет }}=\frac{\pi^{(n-1) / 2} 2^{\alpha+n-1}}{(i \varepsilon)^{\alpha+n}} \sum_{l, j} \frac{(-1)^{l}}{\varepsilon^{2 l}} \theta_{2 l, j} Y_{2 l, j}(\xi)\left(1-\frac{1 / \varepsilon^{2}}{1 / \varepsilon^{2}+1}\right)^{l+(\alpha+n) / 2} . \\
& \cdot \frac{\Gamma(l+(\alpha+n) / 2) \Gamma(l+(\alpha+n+1) / 2)}{\Gamma\left(l+\frac{n}{2}\right)} . \\
& \cdot{ }_{2} F_{1}\left(l+\frac{\alpha+n}{2}, l-\frac{\alpha+1}{2} ; l+\frac{n}{2} ; \frac{1}{1+\varepsilon^{2}}\right) .
\end{aligned}
$$

Проводя преобразования, получаем справедливое при $\varepsilon \geqslant 0$ выражение для $I_{\text {чет }}$ :

$$
\begin{aligned}
& I_{\text {чет }}=\frac{\pi^{(n-1) / 2} 2^{\alpha+n-1}}{(i \varepsilon)^{\alpha+n}} \sum_{l, j} \frac{(-1)^{l}}{\varepsilon^{2 l}} \theta_{2 l, j} Y_{2 l, j}(\xi)\left(\frac{1}{1+\varepsilon^{2}}\right)^{l+(\alpha+n) / 2} . \\
& \cdot \frac{\Gamma(l+(\alpha+n) / 2) \Gamma(l+(\alpha+n+1) / 2)}{\Gamma\left(l+\frac{n}{2}\right)} . \\
& \cdot{ }_{2} F_{1}\left(l+\frac{\alpha+n}{2}, l-\frac{\alpha+1}{2} ; l+\frac{n}{2} ; \frac{1}{1+\varepsilon^{2}}\right) .
\end{aligned}
$$


Переходя к пределу при $\varepsilon \rightarrow 0$, будем иметь

$$
\begin{gathered}
\lim _{\varepsilon \rightarrow 0} I_{\text {чет }}=\frac{\pi^{(n-1) / 2} 2^{\alpha+n-1}}{i^{\alpha+n}} \sum_{l, j}(-1)^{l} \theta_{2 l, j} Y_{2 l, j}(\xi) \cdot \\
\cdot \frac{\Gamma((\alpha+n+l) / 2) \Gamma((\alpha+n+l+1) / 2)}{\Gamma\left(l+\frac{n}{2}\right)} . \\
\cdot{ }_{2} F_{1}\left(l+\frac{\alpha+n}{2}, l-\frac{\alpha+1}{2} ; l+\frac{n}{2} ; 1\right) .
\end{gathered}
$$

Применим формулу для значения гипергеометрической функции в единице (см. (9.122.1) в [3]). После преобразований, получаем

$$
\lim _{\varepsilon \rightarrow 0} I_{\text {чет }}=\frac{\pi^{(n-1) / 2} 2^{\alpha+n-1}}{i^{\alpha+n}} \sum_{l, j}(-1)^{l} \frac{\Gamma(l+(\alpha+n) / 2)}{\Gamma\left(l-\frac{\alpha}{2}\right)} \theta_{2 l, j} Y_{2 l, j}(\xi) .
$$

Теперь перейдем к вычислению суммы ряда в $I$ по нечетным $k$ :

$$
I_{\text {нечет }}=-\frac{\pi^{n / 2}}{(i \varepsilon)^{\alpha+n}} \sum_{k=0}^{\infty} \sum_{l, j} \theta_{2 l, j} Y_{2 l, j}(\xi) \frac{2^{-2 k-1} \Gamma(2 k+\alpha+n+1)}{(i \varepsilon)^{2 k+1} \Gamma\left(k+l+\frac{n+1}{2}\right) \Gamma\left(k-l+\frac{3}{2}\right)} .
$$

Применим формулу для гамма-функции двойного аргумента

$$
I_{\text {нечет }}=-\frac{\pi^{(n-1) / 2}}{(i \varepsilon)^{\alpha+n}} \sum_{k=0}^{\infty} \sum_{l, j} \theta_{2 l, j} Y_{2 l, j}(\xi) \frac{2^{\alpha+n-1} \Gamma\left(k+\frac{\alpha+n+1}{2}\right) \Gamma\left(k+\frac{\alpha+n}{2}+1\right)}{(i \varepsilon)^{2 k+1} \Gamma\left(k+l+\frac{n+1}{2}\right) \Gamma\left(k-l+\frac{3}{2}\right)} .
$$

Для применения формулы гипергеометрического ряда добавим в числитель и знаменатель множители $\Gamma(k+1)$ и поменяем местами знаки суммирования (обоснование ниже):

$$
\begin{aligned}
& I_{\text {нечет }}=\frac{2^{\alpha+n-1} \pi^{(n-1) / 2} i}{(i \varepsilon)^{\alpha+n} \varepsilon} \sum_{l, j} \theta_{2 l, j} Y_{2 l, j}(\xi) \\
& \cdot \sum_{k=0}^{\infty} \frac{\Gamma\left(k+\frac{\alpha+n+1}{2}\right) \Gamma\left(k+\frac{\alpha+n}{2}+1\right) \Gamma(k+1)}{(i \varepsilon)^{2 k} \Gamma\left(k+l+\frac{n+1}{2}\right) \Gamma\left(k-l+\frac{3}{2}\right) \Gamma(k+1)} .
\end{aligned}
$$

При $\varepsilon>1$ ряд по $k$ является обобщенным гипергеометрическим. Он равномерно и абсолютно сходится, когда модуль аргумента меньше единицы. Используя обозначение гипергеометрического ряда, получаем

$$
\begin{aligned}
I_{\text {нечет }}= & \frac{2^{\alpha+n-1} \pi^{(n-1) / 2} i}{(i \varepsilon)^{\alpha+n} \varepsilon} \frac{\Gamma\left(\frac{\alpha+n+1}{2}\right) \Gamma\left(\frac{\alpha+n}{2}+1\right)}{\Gamma\left(l+\frac{n-1}{2}\right) \Gamma\left(\frac{3}{2}-l\right)} . \\
& \cdot \sum_{l, j} \theta_{2 l, j} Y_{2 l, j}(\xi){ }_{3} F_{2}\left(\frac{\alpha+n+1}{2}, \frac{\alpha+n}{2}+1,1 ; l+\frac{n+1}{2}, \frac{3}{2}-l ;-\frac{1}{\varepsilon^{2}}\right) .
\end{aligned}
$$


Запишем обобщенный ряд с помощью интеграла по двойной петле из (4):

$$
\begin{gathered}
I_{\text {нечет }}=\frac{2^{\alpha+n-1} \pi^{(n-1) / 2} i}{(i \varepsilon)^{\alpha+n} \varepsilon} \frac{\Gamma\left(\frac{\alpha+n+1}{2}\right) \Gamma\left(\frac{\alpha+n}{2}+1\right)}{\Gamma\left(l+\frac{n-1}{2}\right) \Gamma\left(\frac{3}{2}-l\right)} \cdot \sum_{l, j} \theta_{2 l, j} Y_{2 l, j}(\xi) . \\
\cdot \int_{(0)}^{(1)}(1-t)^{l+\frac{\alpha+n}{2}-1}{ }_{2} F_{1}\left(\frac{\alpha+n+1}{2}, \frac{\alpha+n}{2}+1 ; \frac{3}{2}-l ;-\frac{t}{\varepsilon^{2}}\right) d t .
\end{gathered}
$$

Как и выше, осуществим аналитическое продолжение гипергеометрического ряда в комплексную плоскость с разрезом по лучу $(1, \infty)$ с помощью преобразования $w=z /(z-1)$ :

$$
\begin{aligned}
I_{\text {нечет }}=\frac{2^{\alpha+n-1} \pi^{(n-1) / 2} i}{(i \varepsilon)^{\alpha+n} \varepsilon} \frac{\Gamma\left(\frac{\alpha+n+1}{2}\right) \Gamma\left(\frac{\alpha+n}{2}+1\right)}{\Gamma\left(l+\frac{n-1}{2}\right) \Gamma\left(\frac{3}{2}-l\right)} & \\
\cdot \sum_{l, j} \theta_{2 l, j} Y_{2 l, j}(\xi) & \cdot \int_{(0)}^{(1)}(1-t)^{l+\frac{\alpha+n}{2}-1} \frac{(\varepsilon)^{\alpha+n+1}}{\left(t+\varepsilon^{2}\right)^{\frac{\alpha+n+1}{2}}} \cdot \\
& \cdot{ }_{2} F_{1}\left(\frac{\alpha+n+1}{2}, \frac{1}{2}-l-\frac{\alpha+n}{2}+1 ; \frac{3}{2}-l ; \frac{t}{t+\varepsilon^{2}}\right) d t .
\end{aligned}
$$

Перейдем к пределу по $\varepsilon$, т.к. выражение в правой части определено для любого $\varepsilon \geqslant 0$ :

$$
\begin{aligned}
\lim _{\varepsilon \rightarrow 0} I_{\text {нечет }} & =\frac{2^{\alpha+n-1} \pi^{(n-1) / 2} i}{i^{\alpha+n}} \frac{\Gamma\left(\frac{\alpha+n+1}{2}\right) \Gamma\left(\frac{\alpha+n}{2}+1\right)}{\Gamma\left(l+\frac{n-1}{2}\right) \Gamma\left(\frac{3}{2}-l\right)} \\
\cdot \sum_{l, j} \theta_{2 l, j} Y_{2 l, j}(\xi){ }_{2} F_{1}\left(\frac{\alpha+n+1}{2}, \frac{1}{2}-l-\frac{\alpha+n}{2}+1 ; \frac{3}{2}-l ; 1\right) \cdot & \cdot \int_{(0)}^{(1)}(1-t)^{l+\frac{\alpha+n}{2}-1} t^{1-\frac{\alpha+n+1}{2}-1} d t .
\end{aligned}
$$

Применяя формулы для интеграла по двойной петле (3) и для гипергеометрической функции в единице (см. (9.122.1), а также (8.344.3) из [3]), получаем:

$$
\lim _{\varepsilon \rightarrow 0} I_{\text {нечет }}=\frac{2^{\alpha+n-1} \pi^{(n-1) / 2} i}{i^{\alpha+n}} \frac{\sin \pi\left(\frac{\alpha+n}{2}\right)}{\sin \pi\left(\frac{\alpha+n+1}{2}\right)} \cdot \sum_{l, j}(-1)^{l} \frac{\Gamma\left(l+\frac{\alpha+n}{2}\right)}{\Gamma\left(l-\frac{\alpha}{2}\right)} \theta_{2 l, j} Y_{2 l, j}(\xi) .
$$

Преобразуем это выражение, записав по формуле Эйлера степень мнимой единицы

$$
\begin{gathered}
\lim _{\varepsilon \rightarrow 0} I_{\text {нечет }}=\frac{2^{\alpha+n-1} \pi^{(n-1) / 2}\left(\sin \pi\left(\frac{\alpha+n}{2}\right)+i \cos \pi\left(\frac{\alpha+n}{2}\right)\right) \sin \pi\left(\frac{\alpha+n}{2}\right)}{\cos \pi\left(\frac{\alpha+n}{2}\right)} . \\
\cdot \sum_{l, j}(-1)^{l} \frac{\Gamma\left(l+\frac{\alpha+n}{2}\right)}{\Gamma\left(l-\frac{\alpha}{2}\right)} \theta_{2 l, j} Y_{2 l, j}(\xi) .
\end{gathered}
$$


Тогда

$$
\begin{aligned}
& \lim _{\varepsilon \rightarrow 0} I_{\text {нечет }}=\left(\frac{2^{\alpha+n-1} \pi^{(n-1) / 2}\left(\sin \pi\left(\frac{\alpha+n}{2}\right)\right)^{2}}{\cos \pi\left(\frac{\alpha+n}{2}\right)}+i \sin \pi\left(\frac{\alpha+n}{2}\right)\right) \\
& \cdot \sum_{l, j}(-1)^{l} \frac{\Gamma\left(l+\frac{\alpha+n}{2}\right)}{\Gamma\left(l-\frac{\alpha}{2}\right)} \theta_{2 l, j} Y_{2 l, j}(\xi) .
\end{aligned}
$$

После вычислений четной и нечетной сумм рядов (7) и (8) получим их сумму

$$
\begin{aligned}
& L \theta(\tau)|t|^{\alpha}=\frac{1}{|x|^{\alpha+n}} \lim _{\varepsilon \rightarrow 0} I=\frac{1}{|x|^{\alpha+n}}\left(\lim _{\varepsilon \rightarrow 0} I_{\text {нечет }}+\lim _{\varepsilon \rightarrow 0} I_{\text {чет }}\right)= \\
& \quad=\frac{2^{\alpha+n-1} \pi^{(n-1) / 2}}{|x|^{\alpha+n} \cos \pi\left(\frac{\alpha+n}{2}\right)} \cdot \sum_{l, j}(-1)^{l} \frac{\Gamma\left(l+\frac{\alpha+n}{2}\right)}{\Gamma\left(l-\frac{\alpha}{2}\right)} \theta_{2 l, j} Y_{2 l, j}(\xi) .
\end{aligned}
$$

В последнем выражении присутствует мультипликаторный оператор $m_{l}$, причем его спектр имеет порядок

$$
m_{l}=(-1)^{l} \frac{\Gamma\left(l+\frac{\alpha+n}{2}\right)}{\Gamma\left(l-\frac{\alpha}{2}\right)} \sim l^{\alpha+n / 2} \text { при } l \rightarrow \infty .
$$

Так как $\theta(\tau) \in \mathrm{C}^{\infty}\left(S^{n-1}\right)$, то по теореме 4.8 из [6]

$$
\theta_{2 l, j}=O\left(l^{-k}\right), \forall k>0 .
$$

Благодаря этому получим абсолютно и равномерно сходящийся ряд в (9). Это дает обоснование перемены знаков суммирования и интегрирования.

Доказательство теоремы 2. Все произведенные вычисления в теореме 1 остаются в силе. Исследуем на сходимость ряд Фурье-Лапласа в (2).

Необходимо обеспечить достаточный порядок гладкости полученной функции на сфере. Мультипликатор $m_{l}$ соответствует операции дифференцирования на сфере порядка $\alpha+n / 2$ при $\alpha+n / 2>0$ (см. [8]). Если же $\alpha+n / 2<0$, то это соответствует сферическому потенцированию, которое улучшает сходимость ряда. Для того, чтобы ряд в $(9)$ был сходящимся в $L_{2}\left(S^{n-1}\right)$ необходимо потребовать, чтобы $\theta(\tau) \in L_{2}^{r}\left(S^{n-1}\right)$, где $r \geqslant \max (0, \alpha+n / 2)$.

Замечание 2. Отметим, что имеет место теорема вложения (см. [7], [8])

$$
L_{2}^{r}\left(S^{n-1}\right) \subset \mathrm{C}^{k}\left(S^{n-1}\right) \text { при } r \geqslant k+n / 2 .
$$

Таким образом, ряды в (2) абсолютно и равномерно сходятся и их сумма принадлежит пространству $\mathrm{C}^{k}\left(S^{n-1}\right)$, если $r \geqslant k+\alpha+n$.

\section{Заключение}

Преобразование Лапласа имеет широкое применение. Одной из областей, в которой оно используется, являются абелевы и тауберовы теоремы, опирающиеся 
на преобразование Лапласа однородных функций, заданных на остром выпуклом конусе и имеющих вид $\theta(\tau)|t|^{\alpha}, \tau \in \gamma$. Для его вычисления используется Фурье-анализ на единичной сфере, в частности, ряды Фурье-Лапласа. Основным приемом, способствующим получению конечного результата, явилось суммирование интегралов с помощью ядра преобразования Фурье, позволившее построить простое аналитическое продолжение появляющихся при вычислениях гипергеометрических функций.

В статье получены формулы преобразования Лапласа однородных функций, у которых $\theta(\tau)$ принадлежит различным функциональным пространствам на единичной сфере с носителем $\gamma$.

\section{Список литературы}

[1] Архипов С.В. О суммировании по Абелю обратного преобразования Фурье однородных функций в $\mathbb{R}^{n} / /$ Вестник ТвГУ. Серия: Прикладная математика. 2019. № 4. C. 98-107. https://doi.org/10.26456/vtpmk547

[2] Владимиров В.С., Дрожжинов Ю.Н., Завьялов Б.И. Многомерные тауберовы теоремы для обобщенных функций. М.: Наука, 1986. 304 с.

[3] Градштейн И.С., Рыжик И.М. Таблицы интегралов, сумм, рядов и произведений. М.: Физматлит, 1963. 1100 с.

[4] Кратцер А., Франц В. Трансцендентные функции. М.: Из-во иностранной литературы, 1963. 467 с.

[5] Люк Ю. Специальные математические функции и их аппроксимации. М.: Мир, 1980. 608 с.

[6] Самко С.Г. Гиперсингулярные интегралы и их приложения. Ростов: Изд-во Ростов. ун-та, 1984. 208 с.

[7] Самко С.Г. Обобщённые риссовы потенциалы и гиперсингулярные интегралы с однородными характеристиками, их символы и обращение // Труды Математического института им. В.А. Стеклова Академии наук СССР. 1980. Т. 156. C. $152-222$.

[8] Самко С.Г. Сингулярные интегралы по сфере и построение характеристики по символу // Известия высших учебных заведений. Математика. 1983. № 4. C. $28-42$.

[9] Muller C. Spherical Harmonics. Series: Lecture Notes in Mathematics. Vol. 17. Berlin, Heidelberg: Springer-Verlag, 1966. 45 p.

[10] Rubin B. Introduction to Radon Transform: with elements of fractional calculations and harmonic analysis. Cambridge University Press, 2015. 576 p. 


\section{Образец цитирования}

Архипов С.В. О преобразовании Лапласа однородных функций в $\mathbb{R}^{n}$ // Вестник ТвГУ. Серия: Прикладная математика. 2020. №1. С.60-71. https://doi.org/10.26456/vtpmk555

\section{Сведения об авторах}

\section{1. Архипов Сергей Викторович}

доцент кафедры математической статистики и системного анализа Тверского государственного университета.

Россия, 170100, г. Тверь, ул. Желябова, д. З3, ТвГУ. 


\title{
THE LAPLACE TRANSFORM OF THE HOMOGENEOUS FUNCTIONS IN $\mathbb{R}^{n}$
}

\author{
Arkhipov Sergey Viktorovich \\ Associate Professor at the Department of Mathematical Statistics and System \\ Analysis, Tver State University \\ 170100, Russia, Tver, 33 Zhelyabova str., TverSU.
}

\begin{abstract}
Received 20.01.2020, revised 01.03.2020.
In the one-dimensional case the Laplace transform of power functions relates to tabular integrals. The multidimensional analogue of power functions are homogeneous functions $\theta(\tau)|t|^{\alpha}$, where $\alpha$ is the degree of homogeneity and $\theta(\tau)$ is a function on the unit sphere $S^{n-1}$. For the convergence of the integral, it is necessary to consider the region $\gamma$ lying inside some hemisphere. In calculating the Laplace transform of homogeneous functions, it is necessary to derive an explicit representation. This is achieved by using Fourier analysis on the sphere, as well as summing the integrals applying the kernel of the Fourier transform, which allows us to construct a simple analytic continuation of the hypergeometric functions that appear in the calculations. The article obtains the formulas for the Laplace transform of homogeneous functions for which $\theta(\tau)$ belongs to different functional spaces on the unit sphere with support $\gamma$.
\end{abstract}

Keywords: multidimensional Laplace transform, homogeneous functions, spherical harmonics, Fourier-Laplace series.

\section{Citation}

Arkhipov S.V., "The Laplace transform of the homogeneous functions in $\mathbb{R}^{n}$ ", Vestnik TvGU. Seriya: Prikladnaya Matematika [Herald of Tver State University. Series: Applied Mathematics], 2020, № 1, 60-71 (in Russian). https://doi.org/10.26456/vtpmk555

\section{References}

[1] Arkhipov S.V., "The Abel summation of the inverse Fourier transform of the homogeneous functions in $R^{n}$ ", Vestnik TvGU. Seriya: Prikladnaya Matematika [Herald of Tver State University. Series: Applied Mathematics], 2019, № 4, 98-107 (in Russian), https://doi.org/10.26456/vtpmk547.

[2] Vladimirov V.S., Drozzinov Yu.N., Zavialov B.I., Tauberian Theorems for Generalized Functions, Kluwer Academic Publishers, Dordrecht, 1988, 292 pp.

[3] Gradshtejn I.S., Ryzhik I.M., Tablitsy integralov, summ, ryadov i proizvedenij [Table of Integrals, Series, and Products], Fizmatlit Publ., Moscow, 1963 (in Russian), $1100 \mathrm{pp}$. 
[4] Kratzer A., Franz W., Transtsendentnye funktsii [Transcendent functions], Iz-vo inostrannoj literatury, M., 1963 (in Russian), 467 pp.

[5] Luke Yu., Spetsialnye matematicheskie funktsii $i$ ikh approksimatsii [Mathematical functions and their approximations], Mir Publ., Moscow, 1980 (in Russian), 608 pp.

[6] Samko S.G., Gipersingulyarnye integraly $i$ ikh prilozheniya [Hypersingular Integrals and Their Applications], Rostov University Press, Rostov, 1984 (in Russian), 208 pp.

[7] Samko S.G., "Obobshchyonnye rissovy potentsialy i gipersingulyarnye integraly s odnorodnymi kharakteristikami, ikh simvoly i obrashchenie", Trudy Matematicheskogo instituta im. V.A. Steklova Akademii nauk SSSR [ Proceedings of the Steklov Institute of Mathematics], 156 (1980), 152-222 (in Russian).

[8] Samko S.G., "Singular integrals over a sphere and construction of characteristics by a symbol", Izvestiya vysshikh uchebnykh zavedenij. Matematika, 1983, № 4, 2842 (in Russian).

[9] Muller C., Spherical Harmonics. V.17, Lecture Notes in Mathematics, SpringerVerlag, Berlin, Heidelberg, 1966, 45 pp.

[10] Rubin B., Introduction to Radon Transform: with elements of fractional calculations and harmonic analysis, Cambridge University Press, 2015, 576 pp. 